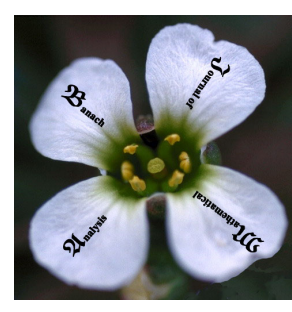

Banach J. Math. Anal. 8 (2014), no. 1, 269-278

B anach Journal of $\mathbf{M}_{\text {athematical }} \mathbf{A}_{\text {nalysis }}$

ISSN: $1735-8787$ (electronic)

www.emis.de/journals/BJMA/

\title{
DETERMINANTAL REPRESENTATION OF TRIGONOMETRIC POLYNOMIAL CURVES VIA SYLVESTER METHOD
}

\author{
MAO-TING CHIEN ${ }^{1 *}$ AND HIROSHI NAKAZATO ${ }^{2}$ \\ Communicated by F. Kittaneh
}

\begin{abstract}
For any trigonometric polynomial $\phi(\theta)$, we give a constructive algorithm by Sylvester elimination which produces matrices $C_{1}, C_{2}, C_{3}$ such that $\operatorname{det}\left(C_{1}+\Re(\phi(\theta)) C_{2}+\Im(\phi(\theta)) C_{3}\right)=0$. For a typical trigonometric polynomial, we assert that $C_{1}$ is positive definite, and thus the typical polynomial curve admits a determinantal representation.
\end{abstract}

\section{INTRODUCTION AND PRELIMINARIES}

Let $A$ be an $n \times n$ matrix. The real ternary form $F_{A}(t, x, y)$ associated to $A$ is defined as

$$
F_{A}(t, x, y)=\operatorname{det}\left(t I_{n}+x \Re(A)+y \Im(A)\right),
$$

where $\Re(A)=\left(A+A^{*}\right) / 2$ and $\Im(A)=\left(A-A^{*}\right) /(2 i)$. Kippenhahn [8] characterized the numerical range of $A, W(A)=\left\{\xi^{*} A \xi: \xi \in \mathbb{C}^{n}, \xi^{*} \xi=1\right\}$, as the convex hull of the real affine part of the dual curve of the curve $F_{A}(t, x, y)=0$. The form $F_{A}(t, x, y)$ is hyperbolic with respect to $(1,0,0)$, i.e., $F_{A}(1,0,0) \neq 0$, and for any real pair $x, y, F_{A}(t, x, y)$ has only real roots in $t$. The converse part was conjectured by Fiedler [5] and Lax [9], namely, for any real ternary hyperbolic form $f(t, x, y)$, there exist Hermitian(or real symmetric) matrices $S_{1}$ and $S_{2}$ such that

$$
f(t, x, y)=\operatorname{det}\left(t I_{n}+x S_{1}+y S_{2}\right)=F_{S}(t, x, y),
$$

Date: Received: 18 March 2013; Accepted: 23 June 2013.

* Corresponding author.

2010 Mathematics Subject Classification. Primary 47A10; Secondary 47A12.

Key words and phrases. Determinantal representation, Sylvester method, numerical range. 
where $S=S_{1}+i S_{2}$. Helton and Vinnikov [6] gave an affirmative answer to the conjecture (see also $[10,12])$. In this case, we call that the form $f(t, x, y)$ admits a determinantal representation by the matrix $S$.

In [2], the authors of this paper study a typical roulette curve given by

$$
\phi(\theta)=\exp (i n \theta)+a \exp (-i(n-1) \theta)
$$

$0 \leq \theta \leq 2 \pi, n=2,3, \ldots$, and $0<a<1$. In particular, they obtain that there exists a $2 n \times 2 n$ matrix $A$ so that the roulette (1.1) is exactly the algebraic curve defined by $F_{A}(t, x, y)$. In other words,

$$
F_{A}(1, \Re(\phi(\theta)), \Im(\phi(\theta)))=0,0 \leq \theta \leq 2 \pi .
$$

A more general form of the roulette curve (1.1) is a class of trigonometric polynomials given by

$$
\phi(\theta)=\sum_{j=-n}^{n} c_{j} \exp (i j \theta) .
$$

The curve $C_{\phi}$ in the Gaussian plane associated to the trigonometric polynomial $\phi$ is defined as

$$
C_{\phi}=\{(\Re(\phi(\theta)), \Im(\phi(\theta))): 0 \leq \theta \leq 2 \pi\} .
$$

By using Henrion method [7] based on Bezoutian resultant, it is shown in [3] that there exist $2 n \times 2 n$ real symmetric matrices $A_{1}, A_{2}, A_{3}$ so that the curve $C_{\phi}$ lies in the curve

$$
\operatorname{det}\left(A_{1}+x A_{2}+y A_{3}\right)=0 .
$$

Sufficient conditions are given in [3] that guarantee the matrix $A_{1}$ being positive definite. In this case, the curve $C_{\phi}$ admits a determinantal representation by the matrix

$$
A_{0}=A_{1}^{-1 / 2}\left(A_{2}+i A_{3}\right) A_{1}^{-1 / 2}
$$

that is $F_{A_{0}}(1, \Re(\phi(\theta)), \Im(\phi(\theta)))=0$.

We continue our study to construct another algorithm, based on Sylvester matrix, that produces matrices $C_{1}, C_{2}, C_{3}$ for trigonometric polynomial $\phi(\theta)$ in (1.3) satisfying

$$
\operatorname{det}\left(C_{1}+\Re(\phi(\theta)) C_{2}+\Im(\phi(\theta)) C_{3}\right)=0 .
$$

For a typical trigonometric polynomial $\phi(\theta)$, we assert that $C_{1}$ is positive definite, and thus the corresponding curve $C_{\phi}$ admits a determinantal representation.

\section{SyLVESTER METHOD}

Consider a complex trigonometric polynomial $\phi(\theta)$ as in (1.3). The conjugate of $\phi(\theta)$ is denoted by

$$
\psi(\theta)=\sum_{j=-n}^{n} \overline{c_{j}} \exp (-i j \theta)=\sum_{j=-n}^{n} \overline{c_{-j}} \exp (i j \theta) .
$$

We substitute the variable $u=\exp (i \theta)$. Then (1.3) and (2.1) respectively become

$$
\sum_{j=-n}^{n} c_{j} u^{n+j}-\phi(\theta) u^{n}=0
$$




$$
\sum_{j=-n}^{n} \overline{c_{-j}} u^{n+j}-\psi(\theta) u^{n}=0
$$

Recall that the $2 \ell \times 2 \ell$ Sylvester matrix $H$ of two polynomials

$$
p(u)=\sum_{j=0}^{\ell} \gamma_{\ell-j} u^{j} \text { and } q(u)=\sum_{j=0}^{\ell} \delta_{\ell-j} u^{j}
$$

is defined as

$$
H=H_{p, q}=\left(\begin{array}{cccccccc}
\gamma_{0} & \gamma_{1} & \ldots & \gamma_{\ell} & 0 & 0 & \ldots & 0 \\
0 & \gamma_{0} & \gamma_{1} & \ldots & \gamma_{\ell} & 0 & \ldots & 0 \\
\vdots & \vdots & \vdots & \ddots & \ddots & \ddots & \ddots & \vdots \\
0 & \ldots & \ldots & \gamma_{0} & \gamma_{1} & \ldots & \ldots & \gamma_{\ell} \\
\delta_{0} & \delta_{1} & \ldots & \ldots & \delta_{\ell} & 0 & \ldots & 0 \\
0 & \delta_{0} & \delta_{1} & \ldots & \ldots & \delta_{\ell} & \ldots & 0 \\
\vdots & \vdots & \vdots & \ddots & \ddots & \ddots & \ddots & \vdots \\
0 & \ldots & \ldots & \delta_{0} & \delta_{1} & \ldots & \ldots & \delta_{\ell}
\end{array}\right)
$$

The determinant of the matrix $H$ is called the resultant of $p(u)$ and $q(u)$ with respect to $u$. It is well known that $p(u)$ and $q(u)$ have a common non-constant factor if and only if $\operatorname{det}(H)=0$ (cf. [4, 13]).

To construct matrices $C_{1}, C_{2}, C_{3}$ satisfying (1.4), we introduce a new parameter $t$ in (2.2) and (2.3), and write

$$
\begin{aligned}
t \sum_{j=-n}^{n} c_{j} u^{n+j}-\phi(\theta) u^{n} & =\sum_{j=0}^{2 n} \gamma_{2 n-j}(t, z) u^{j}, \\
t \sum_{j=-n}^{n} \overline{c_{-j}} u^{n+j}-\psi(\theta) u^{n} & =\sum_{j=0}^{2 n} \delta_{2 n-j}(t, w) u^{j} .
\end{aligned}
$$

Now, let $H$ be the $4 n \times 4 n$ Sylvester matrix of polynomials

$$
p(u: t, z)=\sum_{j=0}^{2 n} \gamma_{2 n-j}(t, z) u^{j} \text { and } q(u: t, z)=\sum_{j=0}^{2 n} \delta_{2 n-j}(t, z) u^{j} .
$$

Denote the matrix $H$ with rows $r_{1}, r_{2}, \ldots, r_{4 n}$ as

$$
H=H\left(r_{1}, r_{2}, \ldots, r_{4 n}\right) .
$$

More precisely, the $j$-th row of the matrix $H$ is

$$
r_{j}=\left(0_{j-1}, c_{n} t, c_{n-1} t, \ldots, c_{0} t-\phi, \ldots, c_{-n} t, 0_{2 n-j}\right)
$$

for $1 \leq j \leq 2 n$, and

$$
r_{j}=\left(0_{j-2 n-1}, \overline{c_{-n}} t, \overline{c_{-n+1}} t, \ldots, \overline{c_{0}} t-\psi, \ldots, \overline{c_{n}} t, 0_{4 n-j}\right)
$$

for $2 n+1 \leq j \leq 4 n$, where $0_{k}$ stands for $k$-dimensional zero vector. We will produce a $2 n \times 2 n$ matrix associated to $\phi(\theta)$ by modifying the matrix $H$. At first, we define the matrix

$$
\tilde{H}=\tilde{H}\left(r_{1}, \ldots, r_{n}, \tilde{r}_{n+1}, \ldots, \tilde{r}_{3 n}, r_{3 n+1}, \ldots, r_{4 n}\right)
$$


which is obtained from $H(2.4)$ by replacing the $n+1, n+2, \ldots, 3 n$ rows with the following new rows

$$
\begin{aligned}
\tilde{r}_{n+1}= & r_{n+1}-c_{-n} / \overline{c_{n}} r_{3 n+1} \\
\tilde{r}_{n+2}= & r_{n+2}-c_{-n} / \overline{c_{n}} r_{3 n+2}-\left(c_{-n+1} \overline{c_{n}}-c_{-n} \overline{c_{n-1}}\right) /{\overline{c_{n}}}^{2} r_{3 n+1} \\
\tilde{r}_{n+3}= & r_{n+3}-c_{-n} / \overline{c_{n}} r_{3 n+3}-\left(c_{-n+1} \overline{c_{n}}-c_{-n} \overline{c_{n-1}}\right) /{\overline{c_{n}}}^{2} r_{3 n+2} \\
& -\left[c_{-n+2}{\overline{c_{n}}}^{2}-c_{-n+1} \overline{c_{n-1}} \overline{c_{n}}+c_{-n}\left({\overline{c_{n-1}}}^{2}-\overline{c_{n-2}} \overline{c_{n}}\right)\right] /{\overline{c_{n}}}^{3} r_{3 n+1}, \\
& \ldots \ldots,
\end{aligned}
$$

and

$$
\begin{aligned}
\tilde{r}_{3 n}= & r_{3 n}-\overline{c_{-n}} / c_{n} r_{n} \\
\tilde{r}_{3 n-1}= & r_{3 n-1}-\overline{c_{-n}} / c_{n} r_{n-1}-\left(c_{n} \overline{c_{-n+1}}-c_{n-1} \overline{c_{-n}}\right) / c_{n}^{2} r_{n} \\
\tilde{r}_{3 n-2}= & r_{3 n-2}-\overline{c_{-n}} / c_{n} r_{n-2}-\left(c_{n} \overline{c_{-n+1}}-c_{n-1} \overline{c_{-n}}\right) / c_{n}^{2} r_{n-1} \\
& -\left[\left(c_{n}^{2} \overline{c_{-n+2}}-c_{n} c_{n-1} \overline{c_{-n+1}}\right)+\overline{c_{-n}}\left(c_{n-1}^{2}-c_{n-2} c_{n}\right)\right] / c_{n}^{3} r_{n} \\
& \ldots \ldots
\end{aligned}
$$

The general rows $\tilde{r}_{n+k}, k=1,2, \ldots, n$, are formulated by

$$
\tilde{r}_{n+k}=r_{n+k}+\sum_{j=1}^{k} \alpha_{j} r_{3 n+k+1-j}
$$

where the coefficients $\alpha_{1}, \alpha_{2}, \ldots, \alpha_{k}$ are uniquely determined so that the $(3 n+1)$ th, $(3 n+2)$-th, $\ldots,(3 n+k)$-th entries of the row $\tilde{r}_{n+k}$ all equal 0 , while the coefficients $\beta_{1}, \beta_{2}, \ldots, \beta_{k}$ of the general rows

$$
\tilde{r}_{3 n+1-k}=r_{3 n+1-k}+\sum_{j=1}^{k} \beta_{j} r_{n+j-k}, k=1, \ldots, n
$$

are uniquely determined so that the $n$-th, $(n-1)$-th, $\ldots,(n-k+1)$-th entries of the row $\tilde{r}_{3 n+1-k}$ equal 0 .

The following result is a key observation for the properties of the matrix $\tilde{H}$ in $(2.5)$.

Theorem 2.1. Let $\tilde{H}$ be the matrix defined in (2.5) corresponding to the trigonometric polynomial $\phi(\theta)$ in (1.3). Then the following hold:

( $i)$ The upper left $n \times n$ principal submatrix of $\tilde{H}$ is an upper triangular matrix with diagonals $\left(c_{n} t, c_{n} t, \ldots, c_{n} t\right)$.

(ii) The lower right $n \times n$ principal submatrix of $\tilde{H}$ is a lower triangular matrix with diagonals $\left(\overline{c_{n}} t, \overline{c_{n}} t, \ldots, \overline{c_{n}} t\right)$.

(iii) The first $n$ entries and the last $n$ entries of the new rows $r_{n+1}^{\sim}, \ldots, r_{2 n}^{\sim}$, $r_{2 n+1}, \ldots, r_{3 n}$ are all 0 .

(iv) The form associated to $\phi(\theta)$ in (1.3) is given by

$$
R(t, x, y) \equiv \operatorname{det}(H)=\operatorname{det}(\tilde{H})=\left|c_{n}\right|^{2 n} t^{2 n} \times \operatorname{det}\left(H_{0}\right),
$$

where $H_{0}$ is the $2 n \times 2 n$ principal submatrix of $\tilde{H}$ by deleting the first $n$ and last $n$ rows and columns. 
(v) If we denote the matrix $H_{0}$ by

$$
H_{0}=H_{0}(t, \phi, \psi)=H_{0}(t, x+i y, x-i y)=t C_{1}+x C_{2}+y C_{3},
$$

then we have

$$
\operatorname{det}\left(C_{1}+\Re(\phi(\theta)) C_{2}+\Im(\phi(\theta)) C_{3}\right)=0 .
$$

The matrix $C_{1}$ obtained in Theorem 2.1 is not necessarily Hermitian and is therefore not positive definite; see, for example, the remark at the end of this section. It is shown in [2] that a special trigonometric polynomial (1.1) admits a determinantal representation. We apply Theorem 2.1 to more general typical trigonometric polynomials of the form $\phi(\theta)=\exp (i n \theta)+a \exp (-i m \theta)$ which guarantee the positive definiteness of $C_{1}$.

Theorem 2.2. Let $\phi(\theta)$ be a trigonometric polynomial defined by

$$
\phi(\theta)=\exp (i n \theta)+a \exp (-i m \theta)
$$

$0 \leq \theta \leq 2 \pi$, where $0<m<n$ are positive integers and $0<a<1$ is a positive real number. Then the matrix $H_{0}=t C_{1}+x C_{2}+y C_{3}$ in (2.7) satisfies the following conditions:

(i) The $2 n \times 2 n$ matrices $C_{1}, C_{2}, C_{3}$ are Hermitian and $C_{1}$ is positive definite.

(ii) The matrix $C_{0}=C_{1}^{-1 / 2}\left(C_{2}+i C_{3}\right) C_{1}^{-1 / 2}$ satisfies

$$
F_{C_{0}}(t, x, y) \operatorname{det}\left(C_{1}\right)=\operatorname{det}\left(H_{0}\right) \text {. }
$$

(iii) For $0 \leq \theta \leq 2 \pi$,

$$
F_{C_{0}}(1, \cos (n \theta)+a \cos (m \theta), \sin (n \theta)-a \sin (m \theta))=0 .
$$

Proof. From (2.7), the matrix $H_{0}(0, x, y)=x C_{2}+y C_{3}$ is the following form

$$
\left(\begin{array}{cc}
0 & P(x, y) \\
Q(x, y) & 0
\end{array}\right)
$$

where $P(x, y)$ is a lower triangular Toeplitz matrix

$$
P(x, y)=\left(\begin{array}{cccc}
p_{1}(x, y) & 0 & 0 & \ldots \\
r p_{2}(x, y) & p_{1}(x, y) & 0 & \ldots \\
r p_{3}(x, y) & p_{2}(x, y) & p_{1}(x, y) & \ldots \\
r \ldots & \ldots & \ldots & \ldots
\end{array}\right) \in M_{n}
$$

with

$$
\begin{aligned}
p_{1}(x, y)= & {\left[\left(-\overline{c_{n}}+c_{-n}\right) x+i\left(-\overline{c_{n}}-c_{-n}\right) y\right] / \overline{c_{n}} } \\
p_{2}(x, y)= & \left(c_{-n+1} \overline{c_{n}}-c_{-n} \overline{c_{n-1}}\right)(x-i y) /{\overline{c_{n}}}^{2} \\
p_{3}(x, y)= & \left\{c_{-n+2}{\overline{c_{n}}}^{2}-c_{-n+1} \overline{c_{n-1}} \overline{c_{n}}+c_{-n}\left({\overline{c_{n-1}}}^{2}-\overline{c_{n-2}} \overline{c_{n}}\right)\right\}(x-i y) /{\overline{c_{n}}}^{3} \\
& \ldots \ldots,
\end{aligned}
$$


and $Q(x, y)$ is an upper triangular Toeplitz matrix

$$
Q(x, y)=\left(\begin{array}{cccc}
q_{1}(x, y) & q_{2}(x, y) & q_{3}(x, y) & \ldots \\
0 & q_{1}(x, y) & q_{2}(x, y) & \ldots \\
0 & 0 & q_{1}(x, y) & \ldots \\
\ldots & \ldots & \ldots & \ldots
\end{array}\right) \in M_{n}
$$

with

$$
\begin{aligned}
q_{1}(x, y)= & {\left[\left(-c_{n}+\overline{c_{-n}}\right) x+i\left(c_{n}+\overline{c_{-n}}\right) y\right] / c_{n}, } \\
q_{2}(x, y)= & {\left[\left(c_{n} \overline{c_{-n+1}}-c_{n-1} \overline{c_{-n}}\right)(x+i y)\right] / c_{n}^{2}, } \\
q_{3}(x, y)= & {\left.\left[\left\{c_{n}^{2} \overline{c_{-n+2}}-c_{n-1} c_{n} \overline{c_{-n+1}}\right)+\overline{c_{-n}}\left(c_{n-1}^{2}-c_{n-2} c_{n}\right)\right\}(x+i y)\right] / c_{n}^{3}, } \\
& \ldots \ldots
\end{aligned}
$$

Hence the matrices $C_{2}, C_{3}$ are Hermitian, and

$$
\begin{aligned}
\operatorname{det}\left(H_{0}(0, x, y)\right)= & \operatorname{det}\left(x C_{2}+y C_{3}\right) \\
= & (-1)^{n} p_{1}(x, y)^{n} q_{1}(x, y)^{n} \\
= & (-1)^{n}\left\{-\overline{c_{n}}(x+i y)+c_{-n}(x-i y)\right\}^{n} \\
& \times\left\{\overline{c_{-n}}(x+i y)-c_{n}(x-i y)\right\}^{n} /\left|c_{n}\right|^{2 n},
\end{aligned}
$$

Let $\ell=n-m$. Then the matrix $C_{1}$ is given by

$$
\left(\begin{array}{ccc}
I_{\ell} & 0_{\ell, 2 n-2 \ell} & a I_{\ell} \\
0_{2 n-2 \ell, \ell} & \left(1-a^{2}\right) I_{2 n-2 \ell} & 0_{2 n-2 \ell, \ell} \\
a I_{\ell} & 0_{\ell, 2 n-2 \ell} & I_{\ell}
\end{array}\right)
$$

which is a real symmetric positive definite matrix. The matrix

$$
C_{0}=C_{1}^{-1 / 2}\left(C_{2}+i C_{3}\right) C_{1}^{-1 / 2}
$$

gives a homogeneous polynomial

$$
F_{C_{0}}(t, x, y)=\operatorname{det}\left(t I_{n}+x C_{1}^{-1 / 2} C_{2} C_{1}^{-1 / 2}+y C_{1}^{-1 / 2} C_{3} C_{1}^{-1 / 2}\right)
$$

satisfying

$$
F_{C_{0}}(t, x, y) \operatorname{det}\left(C_{1}\right)=\operatorname{det}\left(H_{0}\right)=\operatorname{det}\left(t C_{1}+x C_{2}+y C_{3}\right) .
$$

The assertion (iii) follows from the Sylvester construction (2.6) and (2.7) for the trigonometric polynomial $\phi(\theta)$, i.e.,

$$
F_{C_{0}}(1, \Re(\phi(\theta)), \Im(\phi(\theta)))=0,0 \leq \theta \leq 2 \pi .
$$

Remark 2.3. Although the matrix $C_{1}$ in Theorem 2.2 is positive definite for $\phi(\theta)=\exp (i n \theta)+a \exp (-i m \theta)$, in general, $C_{1}$ is not Hermitian for an arbitrary trigonometric polynomial $\phi(\theta)$ given in (1.3). For example, let $n=2$ and

$$
\phi(\theta)=\exp (2 i \theta)-\frac{1}{4} \exp (i \theta)-\frac{17}{72}+\frac{1}{36} \exp (-i \theta)+\frac{1}{72} \exp (-2 i \theta) .
$$

Then

$$
\phi(\theta) \exp (2 i \theta)=\left(\exp (i \theta)+\frac{1}{3}\right)\left(\exp (i \theta)+\frac{1}{4}\right)\left(\exp (i \theta)-\frac{1}{3}\right)\left(\exp (i \theta)-\frac{1}{2}\right) .
$$


The matrices constructed by Theorem 2.2 are

$$
C_{1}=\left(\begin{array}{cccc}
20732 & -5192 & -4828 & 648 \\
-9 & 20714 & -5039 & -4666 \\
-4666 & -5039 & 20714 & -9 \\
r 648 & -4828 & -5192 & 20732
\end{array}\right)
$$

and

$$
x C_{2}+y C_{3}=\left(\begin{array}{cccc}
0 & 0 & \alpha & 0 \\
0 & 0 & \beta & \alpha \\
\bar{\alpha} & \bar{\beta} & 0 & 0 \\
0 & \bar{\alpha} & 0 & 0
\end{array}\right)
$$

where $\alpha=-20448 x-21024 y i, \beta=648 x-648 y i$. The matrix $C_{1}$ is not Hermitian.

\section{Discussion}

Let $0<m<n$ be two positive integers and $0<a<1$ be a real number. Consider a trigonometric polynomial $\phi(\theta)=\exp (i n \theta)+a \exp (-i m \theta), 0 \leq \theta \leq 2 \pi$ which defines a real affine curve by the relation

$$
x=x(\theta)=\Re(\phi(\theta)), y=y(\theta)=\Im(\phi(\theta)),
$$

$0 \leq \theta \leq 2 \pi$. Based on Bezoutian, the authors of this paper [3] gave a constructive proof by providing real symmetric matrices $A_{1}, A_{2}, A_{3}$ so that the curve $(x(\theta), y(\theta))$ lies on $\operatorname{det}\left(A_{1}+x A_{2}+y A_{3}\right)=0$.

We compare the two construction matrices obtained in [3] and Theorem 2.2 by investigating the following example. The relation between Bezoutian and Sylvester resultants can be found in [11]. Let $n=2, m=1, a=4 / 5$,

$$
\phi(\theta)=\exp (2 i \theta)+\frac{4}{5} \exp (-i \theta)
$$

Then the matrix $H_{0}(t, x, y)=t C_{1}+x C_{2}+y C_{3}$ in (2.7) is computed by

$$
\begin{gathered}
C_{1}=\left(\begin{array}{cccc}
1 & 0 & 0 & 4 / 5 \\
0 & 9 / 25 & 0 & 0 \\
0 & 0 & 9 / 25 & 0 \\
4 / 5 & 0 & 0 & 1
\end{array}\right), \\
C_{2}=\left(\begin{array}{cccc}
0 & 0 & -1 & 0 \\
0 & 0 & 4 / 5 & -1 \\
-1 & 4 / 5 & 0 & 0 \\
0 & -1 & 0 & 0
\end{array}\right), C_{3}=\left(\begin{array}{cccc}
0 & 0 & -i & 0 \\
0 & 0 & -4 i / 5 & -i \\
i & 4 i / 5 & 0 & 0 \\
0 & i & 0 & 0
\end{array}\right) .
\end{gathered}
$$

We have that

$$
\left(C_{1}\right)^{-1 / 2} C_{2}\left(C_{1}\right)^{-1 / 2}=\frac{5}{9}\left(\begin{array}{cccc}
0 & \sqrt{5} & -2 \sqrt{5} & 0 \\
\sqrt{5} & 0 & 4 & -2 \sqrt{5} \\
-2 \sqrt{5} & 4 & 0 & \sqrt{5} \\
0 & -2 \sqrt{5} & \sqrt{5} & 0
\end{array}\right)
$$


and

$$
\left(C_{1}\right)^{-1 / 2} C_{3}\left(C_{1}\right)^{-1 / 2}=\frac{5}{9}\left(\begin{array}{cccc}
0 & -i \sqrt{5} & -2 i \sqrt{5} & 0 \\
i \sqrt{5} & 0 & -4 i & -2 i \sqrt{5} \\
2 i \sqrt{5} & 4 i & 0 & -i \sqrt{5} \\
0 & 2 i \sqrt{5} & i \sqrt{5} & 0
\end{array}\right) .
$$

Thus the matrix $C_{0}=C_{1}^{-1 / 2}\left(C_{2}+i C_{3}\right) C_{1}^{-1 / 2}$ in Theorem 2.2 is given by

$$
C_{0}=\frac{10}{9}\left(\begin{array}{cccc}
0 & \sqrt{5} & 0 & 0 \\
0 & 0 & 4 & 0 \\
-2 \sqrt{5} & 0 & 0 & \sqrt{5} \\
0 & -2 \sqrt{5} & 0 & 0
\end{array}\right)
$$

On the other hand, the matrices constructed by Bezoutian in [3] satisfying

$$
6250000 \operatorname{det}\left(t C_{1}+x C_{2}+y C_{3}\right)=\operatorname{det}\left(t A_{1}+x A_{2}+y A_{3}\right)
$$

are given by

$$
A_{1}=\left(\begin{array}{cccc}
27 & 0 & -63 & 0 \\
0 & 27 & 0 & -3 \\
-63 & 0 & 207 & 0 \\
0 & -3 & 0 & 7
\end{array}\right)
$$

and

$$
A_{2}=\left(\begin{array}{cccc}
-15 & 0 & 35 & 0 \\
0 & 65 & 0 & 15 \\
35 & 0 & 85 & 0 \\
0 & 15 & 0 & -35
\end{array}\right), A_{3}=\left(\begin{array}{cccc}
0 & -60 & 0 & -10 \\
-60 & 0 & -10 & 0 \\
0 & -10 & 0 & 40 \\
-10 & 0 & 40 & 0
\end{array}\right)
$$

The matrix $A_{1}^{-1 / 2}$ is a scalar multiple of the matrix

$$
S=\left(\begin{array}{cccc}
p & 0 & q & 0 \\
0 & u & 0 & v \\
q & 0 & r & 0 \\
0 & v & 0 & w
\end{array}\right)
$$

where

$$
\begin{aligned}
& p=\sqrt{218(6217+98 \sqrt{5})}, q=7 \sqrt{218(13-2 \sqrt{5})}, r=\sqrt{218(257+98 \sqrt{5})} \\
& u=\sqrt{298(1373+54 \sqrt{5})}, v=3 \sqrt{298(17-6 \sqrt{5})}, \quad w=3 \sqrt{298(637+6 \sqrt{5})}
\end{aligned}
$$

More precisely $S=2 \sqrt{108} \sqrt{149} A_{1}^{-1 / 2}$.

The matrices $A_{1}^{-1 / 2} A_{2} A_{1}^{-1 / 2}$ and $A_{1}^{-1 / 2} A_{3} A_{1}^{-1 / 2}$ are respectively real symmetric matrices of the form

$$
\left(\begin{array}{cccc}
a_{11} & 0 & a_{13} & 0 \\
0 & a_{22} & 0 & a_{24} \\
a_{13} & 0 & a_{33} & 0 \\
0 & a_{24} & 0 & a_{44}
\end{array}\right) \text { and }\left(\begin{array}{cccc}
0 & a_{12} & 0 & a_{14} \\
a_{12} & 0 & a_{23} & 0 \\
0 & a_{23} & 0 & a_{34} \\
a_{14} & 0 & a_{34} & 0
\end{array}\right)
$$

where $a_{i j}$ 's are distinct non-zero real numbers. Therefore none of entries of the matrix $A_{0}=A_{1}^{-1 / 2}\left(A_{2}+i A_{3}\right) A_{1}^{-1 / 2}$ is 0 , while the matrix $C_{0}$ in (3.1) obtained by 
Theorem 2.2 is rather sparse. The sparsity of $A_{0}$ and $C_{0}$, obtained by the two methods, is an interesting subject for further study.

We have proposed two constructive algorithms for determinantal representations of the trigonometric polynomial $\phi(\theta)=\exp (i n \theta)+a \exp (-i m \theta)$ by matrices $A_{0}=A_{1}^{-1 / 2}\left(A_{2}+i A_{3}\right) A_{1}^{-1 / 2}$ and $C_{0}=C_{1}^{-1 / 2}\left(C_{2}+i C_{3}\right) C_{1}^{-1 / 2}$ satisfying (1.2). It is interesting to ask whether the two matrices $A_{0}$ and $C_{0}$ are unitarily similar. At this time, we cannot answer this question. Nevertheless, we give a positive answer for the case when

$$
\phi(\theta)=\exp (2 i \theta)+4 / 5 \exp (-i \theta) .
$$

According to [2], there constructs a matrix

$$
B=\frac{10}{9}\left(\begin{array}{cccc}
0 & -4 & 0 & 0 \\
0 & 0 & -4 & -3 \\
-5 & 0 & 0 & 0 \\
0 & 0 & 0 & 0
\end{array}\right)
$$

satisfying

$$
729 \operatorname{det}\left(t I_{4}+x \Re(B)+y \Im(B)\right)=15625 \operatorname{det}\left(t C_{1}+x C_{2}+y C_{3}\right) .
$$

At first, we show that the matrices $A_{0}$ and $B$ are unitarily similar by a unitary intertwining matrix $W$ :

$$
W A_{1}^{-1 / 2}\left(A_{2}+i A_{3}\right) A_{1}^{-1 / 2}=B W .
$$

Setting $W A_{1}^{1 / 2}=V$, the matrix $V$ satisfies

$V A_{1}^{-1}\left(A_{2}+i A_{3}\right)=W A_{1}^{1 / 2} A_{1}^{-1}\left(A_{2}+i A_{3}\right)=W A_{1}^{-1 / 2}\left(A_{2}+i A_{3}\right)=B W A_{1}^{1 / 2}=B V$,

and

$$
V A_{1}^{-1} V^{*}=W A_{1}^{1 / 2} A_{1}^{-1} A_{1}^{1 / 2} W^{*}=W W^{*}=I_{4} .
$$

Conversely, if $V$ satisfies (3.2) and (3.3) then the unitary matrix $W=V A_{1}^{-1 / 2}$ satisfies $W A_{1}^{-1 / 2}\left(A_{2}+i A_{3}\right) A_{1}^{-1 / 2} W^{*}=B$. Such a matrix $V$ is given by

$$
V=\left(\begin{array}{cccc}
-3 i / 2 & 3 / 2 & -3 i / 2 & 3 / 2 \\
3 i / 2 & 3 / 2 & 3 i / 2 & 3 / 2 \\
-3 i / 2 & -9 / 2 & 9 i / 2 & 3 / 2 \\
9 i / 2 & -3 / 2 & -27 i / 2 & 1 / 2
\end{array}\right)
$$

This shows that $A_{0}$ and $B$ are unitarily similar.

On the other hand, the matrix $C_{0}$ is unitarily similar to $B$, and $U C_{0} U^{*}=B$ for the unitary matrix

$$
U=\left(\begin{array}{cccc}
0 & -1 & 0 & 0 \\
0 & 0 & 1 & 0 \\
1 / \sqrt{5} & 0 & 0 & -2 / \sqrt{5} \\
2 / \sqrt{5} & 0 & 0 & 1 / \sqrt{5}
\end{array}\right)
$$

Thus, both $A_{0}$ and $C_{0}$ are unitarily similar to $B$. 
Acknowledgement. The authors thank the anonymous referee for his/her careful reading and valuable suggestions. The first author was partially supported by Taiwan National Science Council under NSC101-2115-M-031-002. The second author was supported in part by Japan Society for Promotion of Science, Project Number 23540180.

\section{REFERENCES}

1. E. Bézout, General Theory of Algebraic Equations, Princeton University Press, Princeton and Oxford, 2006. (Tralslared from the French by E. Feron)

2. M.T. Chien and H. Nakazato, Numerical range for orbits under a central force, Math. Phys. Anal. Geom. 13 (2010), 315-330.

3. M.T. Chien and H. Nakazato, Construction of determinantal representation of trigonometric polynomials, Linear Algebra Appl. 435 (2011), 1277-1284.

4. M.T. Chien, H. Nakazato and P. Psarrakos, Point equation of the boundary of the numerical range of a matrix polynomial, Linear Algebra Appl. 347 (2002), 205-217.

5. M. Fiedler, Geometry of the numerical range of matrices, Linear Algebra Appl. 37 (1981), $81-96$.

6. J.W. Helton and V. Vinnikov, Linear matrix inequality representations of sets, Comm. Pure Appl. Math. 60 (2007), 654-674.

7. D. Henrion, Detecting rigid convexity of bivariate polynomials, Linear Algebra Appl. 432 (2010), 1218-1233.

8. R. Kippenhahn, Über den wertevorrat einer Matrix, Math. Nachr. 6(1951), 193-228.

9. P.D. Lax, Differential equations, difference equations and matrix theory, Comm. Pure Appl. Math. 6 (1958), 175-194.

10. A.S. Lewis, P.A. Parrilo and M.V. Ramana, The Lax conjecture is true, Proc. Amer. Math. Soc. 133 (2005), 2495-2499.

11. M. Mignotte, Mathematics for Computer Algebra, Springer, New York, 1992.

12. D. Plaumann, B. Sturmfels, and C. Vinzant, Computing linear matrix representations of Helton-Vinnikov curves, arXiv:1011.6057v1, 2010.

13. R.J. Walker, Algebraic curves, Dover Publ., New York, 1950.

14. H.K. Wimmer, On the history of the Bezoutian and the resultant matrix, Linear Algebra Appl. 128 (1990), 27-34.

1 Department of Mathematics, Soochow University, Taipei 11102, Taiwan.

E-mail address: mtchien@scu.edu.tw

2 Department of Mathematical Sciences, Faculty of Science and Technology, Hirosaki University, Hirosaki 036-8561, JAPAN.

E-mail address: nakahr@cc.hirosaki-u.ac.jp 